\title{
Comparative Analysis of QoS Routing Protocols in MANETS: Unicast \& Multicast
}

\author{
Surabhi Gupta ${ }^{1}$, Manish Saxena ${ }^{2}$ \\ Electronics \& Communication Department, Bansal Institute of Science \& Technology, Bhopal, M.P, India
}

\begin{abstract}
In mobile ad hoc networks (MANETs), the provision of quality of service (QoS) guarantees is much more challenging than in wire line networks, mainly due to node mobility, multi hop communications, contention for channel access, and a lack of central coordination. The difficulties in the provision of such guarantees have limited the usefulness of MANETs. in the last decade, much research attention has focused on providing QoS assurances in MANET protocols. In this paper we have analysed different types of routing protocols and QoS metrics in MANETS.
\end{abstract}

Keywords: MANETS, Qos, unicast routing protocols, multicast Routing protocols

\section{Introduction}

Wireless communication technology have been developed with two primary models one is fix infrastructure based model in which much of the nodes are mobile and connected through fixed backbone nodes using wireless medium. Another model is Mobile Ad-hoc network .Mobile Ad-Hoc Networks (MANETs) are comprised of mobile nodes (MNs) that are self-organizing and cooperative to ensure efficient and accurate packet routing between nodes (and, potentially, base stations).Fig1. shows the structure of MANETS. There are no specific routers, servers, access points for MANETs. Because of its fast and easy of deployment, robustness, and low cost, Typical MANETs applications could be find in the following areas like Military applications (i.e. a temporary network in the battlefield), Search and rescue operations, Temporary networks within meeting rooms, airports, Vehicle-to-vehicle communication in smart transportation, Personal Area Networks connecting mobile devices like mobile phones, laptops, smart watches, and other wearable computers etc. Design issue for developing a routing protocol for wireless environment with mobility is very different and more complex than those for wired network with static nodes [1]. Main problem in mobile ad-hoc network are limited bandwidth and frequent changes in the topology.

Routing protocols for MANETs could be differ depending on the application and network architecture. Routing protocols typically fall under two classifications, first one is Unicast routing protocol, second one is Multicast routing protocol [4].Different routing protocols try to solve the problem of routing in mobile ad hoc network in one way or the other. It observes that any one protocol cannot fit in all the different scenarios, different topologies and traffic patterns of Mobile Ad-Hoc Networks applications. For instance, proactive routing protocols are very useful for a small scale MANETs with high mobility, while reactive routing protocols are very useful for a large-scale, MANETs with moderate or less topology changes. Hybrid routing protocol attempts to strike balance between the two such as proactive for neighborhood, reactive for far away

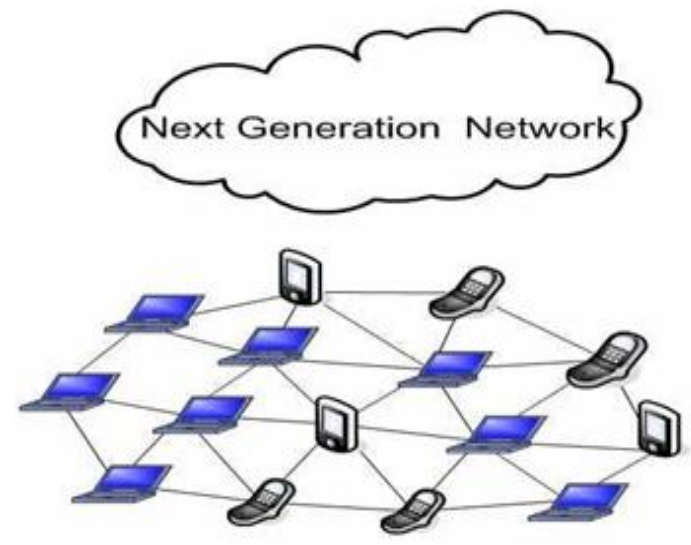

Figure 1: Mobile Ad Hoc Networks-MANETs

In mobile ad hoc networks (MANETs), the provision of quality of service (QoS) guarantees is much more challenging than in wire line networks, mainly due to node mobility, multi hop communications, contention for channel access, and a lack of central coordination.

This paper is organised as follows: In section II, Qos metrics in MANETS are discussed, In section III, various Unicast routing protocols are discussed, In section IV, Multicast routing protocols and are discussed and this paper is routing protocols and are discussed and this paper is concluded in section $\mathrm{V}$.

\section{QOS and Routing Protocols in Manets}

\section{A. Quality of service}

Quality of Service (QoS) means that the network should provide some kind of guarantee or assurance about the level or grade of service provided to an application. The actual form of QoS and the QoS parameter to be considered depends upon specific requirements of an application. For example, an application that is delay sensitive may require the QoS in terms of delay guarantees. Some applications may require that the packets should flow at certain minimum bandwidth. In that case, the bandwidth will be a QoS parameter. Certain application may require a guarantee that the packets are delivered from a given source to destination reliably, then, reliability will be a parameter for QoS. 


\section{International Journal of Science and Research (IJSR) \\ ISSN (Online): 2319-7064}

Index Copernicus Value (2013): 6.14 | Impact Factor (2014): 5.611

\section{B. Metrics used to specify QoS requirements}

The following is a sample of the metrics commonly used by applications to specify QoS requirements to the routing protocol. Consequently, they may be used as constraints on route discovery and selection. Each metric is followed by a reference which provides an example of a protocol that employs the metric as a QoS constraint.

- Minimum Required Throughput or Capacity (b/s) - the desired application data throughput. For an example of QoS routing using this metric/constraint [5].

- Maximum Tolerable Delay (s) - usually defined as the maximum tolerable end-to-end (source to destination) delay for data packets [6].

- Maximum Tolerable Delay Jitter — one widely accepted definition of this metric is the difference between the upper bound on end-to-end delay and the absolute minimum delay [7]. The former incorporates the queuing delay at each node and the latter is determined by the propagation delay and the transmission time of a packet. The transmission time between two nodes is simply the packet size in bits/the channel capacity. This metric can also be expressed as delay variance [8].

- Maximum Tolerable Packet Loss Ratio (PLR) - the acceptable percentage of total packets sent, which are not received by the transport or higher layer agent at the

\section{Routing protocols}

In [3], QoS routing protocols are classified chiefly by their

- Treatment of network topology (flat, hierarchical, or location-aware)

- Approach to route discovery (proactive, reactive, hybrid, or predictive)

\section{Pro-active routing protocols}

In proactive routing protocols, each node maintains routing information to every other node in the network. The routing information is usually kept in a number of different tables. These tables are periodically updated and/or if the network topology changes. Each routing protocol may maintain different number of tables. Pro active routing protocols are also known as Table-driven protocols.

\section{E. Reactive routing protocols}

Reactive routing protocols are designed to reduce the overheads in pro active protocols by maintaining information for active routes only. The routes are determined and maintained for nodes that require to send data to a particular destination. Reactive routing protocols are also known as on-demand routing protocols.

\section{F. Hybrid routing protocols}

Hybrid routing protocols are a new generation of protocol, which are both proactive and reactive in nature. These protocols are designed to increase scalability by allowing nodes with close proximity to work together to form some sort of a backbone to reduce the route discovery overheads.

\section{Unicast Routing Protocols}

All LANs support unicast transfer mode and most applications that employ TCP transport protocol uses unicast messaging. Most applications in the MANET are based upon uni-cast communication. Figure 3 shows the uni-cast process. In the uni-cast routing one separate copy sends to each receiver from the source node. Data packet is replicated at the sender node (BBC server) and then delivered to each destination node. By this process we can easily see that bandwidth is consumed by the redundant data packets.

Many applications use the uni-cast routing protocol depending upon the need of the application. There are proactive, reactive and hybrid routing protocol in uni-cast routing for Mobile distributed networks.

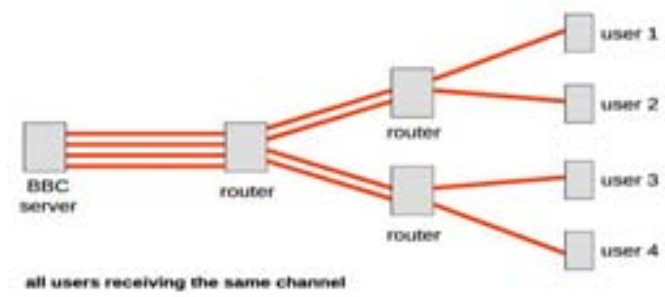

Figure 3: Unicast Process

\section{A. Proactive Unicast Routing Protocols}

Traditional routing protocols such as Optimized link state routing protocol (OLSR), The Fisheye State Routing (FSR), And Topology Broadcast Based on Reverse-Path Forwarding Routing Protocol (TBRPF) are proactive unicast routing protocols. Periodic broadcast of network topology updates (e.g., distance vector or link state information) is necessary to compute the shortest path from the source to every destination, which consumes a lot of bandwidth. Although they are widely used in the Internet backbone. They cannot be used in the MANET directly because of the differences between the hardwired network and the MANET. In Table 1 gives the Characteristic comparison of proactive Unicast Routing Protocol[4].

\section{Optimized Link State Routing Protocol (OLSR)}

Optimized link state routing protocol (OLSR)[10] is a proactive (table-driven) routing protocol for MANETs. A route between source to destination is available immediately when needed. OLSR is based on the link-state algorithm. OLSR used hop-by-hop routing. Routes are based on dynamic table entries maintained at intermediate nodes. Advantage of OLSR is having the routes available within the standard outing table can be useful for some systems and network applications as there is no route discovery delay associated with finding a new route. Bigger overhead and need more power are main disadvantage of this protocol.

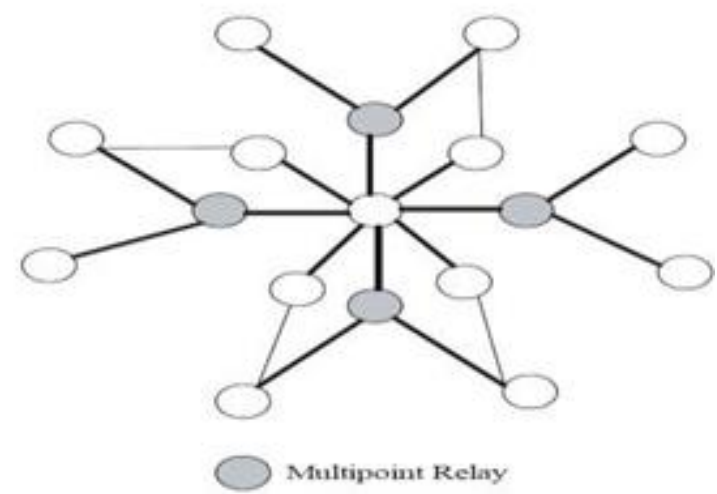

Figure 4: Multi point Relays in OLSR 


\section{International Journal of Science and Research (IJSR) \\ ISSN (Online): 2319-7064}

Index Copernicus Value (2013): 6.14 | Impact Factor (2014): 5.611

\section{Fisheye State Routing Protocol (FSR)}

The Fisheye State Routing (FSR) [11] is a table driven unicast routing protocol for Mobile Ad hoc Networks based on Link State routing algorithm in effect with reduced overhead to keep network topology information. As showed in its name, FSR utilizes a function similar to a fish eye. Advantage of this protocol is that it has potentiality to support multiple-path routing and QoS routing but disadvantage of FSR is that it has high storage complexity.

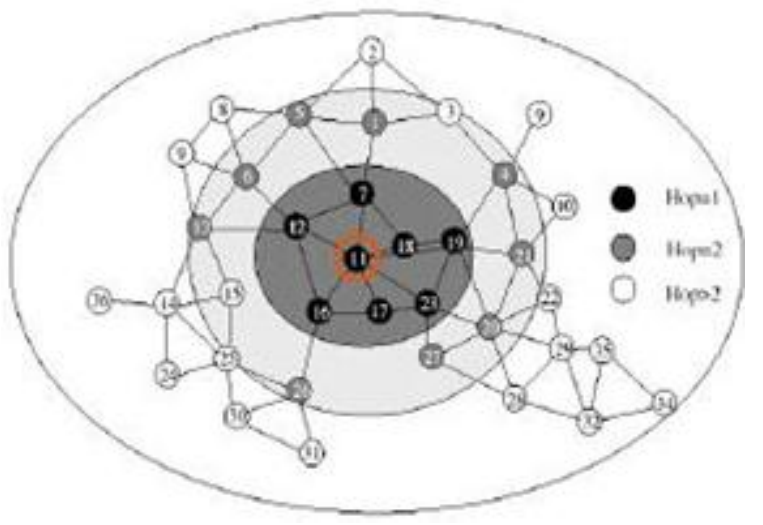

The scope of fisheye is defined as the set of nodes that can be reached within a given number of hops from a particular center node. In the figure 5, we have shown three scopes with one, two, and three hops. The center node has the most accurate information about all nodes in the white circle and so on. Each circle contains the nodes of a particular hop from a center node. Advantage of this protocol is that it has potentiality to support multiple-path routing and QoS routing but disadvantage of FSR is that it has high storage complexity.

\section{Topology Broadcast Based on Reverse-Path Forwarding Routing Protocol (TBRPF)}

Topology Broadcast Based on Reverse-Path Forwarding Routing Protocol (TBRPF) was proposed in [12]. TBRPF aims at the Mobile Ad hoc Network with at most several hundreds of mobile nodes or high mobility of nodes. Every node in the wireless network keeps partial global topology information. When a node needs the shortest path to every other node, a minimum spanning tree rooted at itself is computed using modified Dijkstra's algorithm. TBRPF transmits only the differences between the previous network state and the current network state. Therefore, routing messages are smaller, and can therefore be sent more frequently. This means that nodes' routing tables are more up-to-date.

\section{B. Reactive Uni-cast Routing Protocols}

To minimizing the wastage of bandwidth, the concept of On Demand or reactive routing protocol is proposed in [13].In On Demand protocols, the routing is divided into the following two steps: first one is route discovery and second one is route maintenance. The most distinctive On Demand unicast routing protocols are Dynamic Source Routing (DSR) protocol, Ad Hoc On demand Distance Vector Routing (AODV) protocol and Temporally Ordered Routing Algorithm(TORA) etc. Table 2 gives the characteristic comparison of Reactive Unicast Routing Protocols[4].

\section{Dynamic Source Routing (DSR)}

Dynamic Source Routing (DSR) [14] is an On Demand unicast routing protocol that utilizes source routing algorithm. In source routing algorithm, each data packet contains complete routing information to reach its dissemination. Additionally, in DSR each node uses caching technology to maintain route information. For example, the intermediate nodes cache the route towards the destination and backward to the source. Furthermore, because the data packet contains the source route in the header, the overhearing nodes are able to cache the route in its routing cache.

\section{Ad Hoc On-demand Distance Vector Routing Protocol (AODV)}

The Ad Hoc On-demand Distance Vector Routing (AODV) protocol [15] is a reactive unicast routing protocol for mobile ad hoc networks. As a reactive routing protocol, AODV only needs to maintain the routing information about the active paths. In AODV, routing information is maintained in routing tables at nodes. Every mobile node keeps a next-hop routing table, which contains the destinations to which it currently has a route A routing table entry expires if it has not been used or reactivated for a prespecified expiration time. Moreover, AODV adopts the destination sequence number technique used by DSDV in an on-demand way.

\section{Temporally Ordered Routing Algorithm}

Temporally Ordered Routing Algorithm (TORA) $[16,17]$ is a On Demand routing algorithm based on the concept of link reversal. The Routing protocol improves the partial link reversal method by detecting partitions and stopping nonproductive link reversals. TORA can be used for highly dynamic mobile ad hoc networks. TORA has three basic steps: route creation, route maintenance and route erasure. In TORA the Directed Acyclic Graph (DAG) provides the capability that many nodes can send packets to a given destination and guarantees that all routes are loop-free. Because of node mobility the DAG in TORA may be disconnected. So, route maintenance step is an very important in TORA which has the unique feature that control messages are localized into a small set of nodes near the topology changes occurred.

\section{Multicast Routing Protocols}

Hybrid routing protocol attempts to discover balance between the two such as proactive for neighbor hood, reactive for far away. Based on proactive and reactive routing protocols, some hybrid routing protocols are proposed to combine their advantages. The most distinctive hybrid routing protocol is Zone Routing Protocol.

\section{1) Zone Routing Protocol (ZRP)}

Multicasting can be defined as the process of the concurrently transmission of the same single copy of data packets to several destinations which they identified by a single address. The transmitter may be one or multiple nodes. The former is called one to many model but the latter is called many to many model.

Compared to unicast routing protocols, there are relatively

\section{Volume 4 Issue 11, November 2015}




\section{International Journal of Science and Research (IJSR) \\ ISSN (Online): 2319-7064}

Index Copernicus Value (2013): 6.14 | Impact Factor (2014): 5.611

few multicast routing protocols. Supporting multicast service is not easy even in wired networks. Group management will be very challenging in mobile environment. The cost involved in dynamic group management may make most proposals infeasible for wireless mobile networks.

Although multicast transmission has not been widely deployed in the current MANETs, it will become very important in multimedia communications in the near future. To send a same data packet to multiple receivers in the MANET simultaneously, the simplest method is to broadcast the data packets. However, broadcast consumes considerable bandwidth and power, which should be avoided as much as possible. Multicast can be used for saving the bandwidth while transmitting same data packets to multiple receivers. Figure 6 shows the multicast process, data packet is replicated by the network there have been many multicast routing protocols proposed for MANET. They could be divided into three groups: first one is proactive multicast, second one is reactive multicast and last one is hybrid multicast routing protocol.

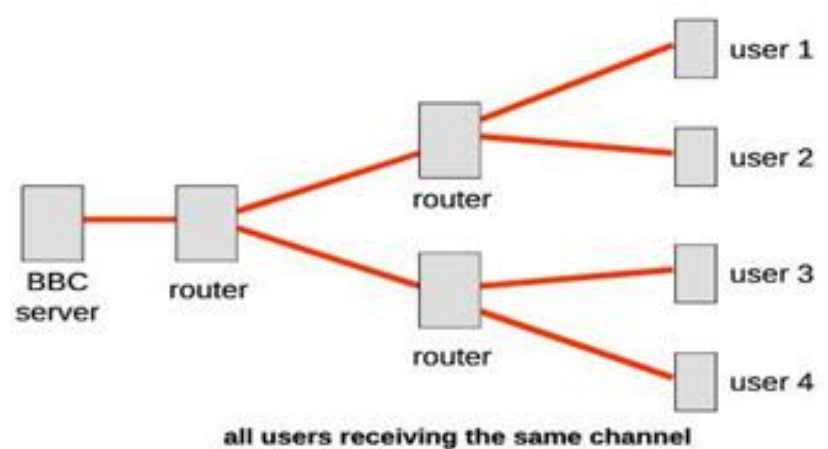

Figure 6: Multicast Process

\section{A. Proactive Multicast Routing Protocols}

Conventional routing protocols such as Ad-hoc Multicast Routing (AM Route), and Ad-hoc Multicast Routing Protocol Utilizing Increasing id-numbers (AMRIS) are proactive multicast routing protocols. Periodic broadcast of network topology updates are needed to compute the shortest path from the source to every destination, which consumes a lot of bandwidth. Table 3 gives the Characteristic comparison of proactive Multicast Routing Protocol[4].

\section{1) Ad-hoc Multicast Routing (AM Route)}

Ad-hoc Multicast Routing (AM Route)[19] is a tree based multicast routing protocol for mobile ad hoc networks . AM Route creates a multicast shared-tree over mesh. AM Route relies on the existence of an underlying uni-cast routing protocol. AM Route has two key phases: mesh creation and tree creation. AM Route protocol can be used for networks in which only a set of nodes supports AM Route routing function. It is only one logical core in the multicast tree, which is responsible for group member maintenance and multicast tree creation. AM Route builds a user- multicast tree, in which only the group members are included; because non-members are not included in the tree, the links in the tree are virtual links. In other words, they are in fact multihop IP-in-IP tunnels and AM Route depends on the underlying uni-cast routing protocol to deal with network dynamics, although it has no privilege for uni-cast routing protocols. AM Route creates an efficient and robust shared tree for each group and helps to keep the multicast delivery tree unchanged with changes of network topology, as long as paths between tree members and core nodes exist via mesh links. When mobility is present, AM Route suffers from loop formation, creates non optimal trees, and requires higher overhead to assign a new core. Also, AM Route suffers from a single point of failure of the core node.

Table 3: Characteristics of Proactive Multicast Routing Protocol

\begin{tabular}{|c|c|c|c|}
\hline Characteristics & AMRoute & AMRIS & CAMP \\
\hline Structure of multicast delivery & Tree & Tree & Mesh \\
\hline Loop free & No & Yes & Yes \\
\hline $\begin{array}{c}\text { Dependency on unicast routing } \\
\text { protocol }\end{array}$ & Yes & No & Yes \\
\hline Scalability & Fair & Fair & Good \\
\hline Control packet flooding & Flat & Flat & Flat \\
\hline Periodic message requirement & Yes & Yes & Yes \\
\hline
\end{tabular}

\section{2) Ad hoc Multicast Routing Protocol Utilizing} Increasing id-numbers (AMRIS)

AMRIS [20] is a proactive shared tree based multicast routing protocol, which is independent of the fundamental unicast routing protocol. In AMRIS, the tree maintenance procedure operates continuously and locally to ensure a node's connection to the multicast session delivery tree. In AMRIS, the tree maintenance procedure operates continuously and locally to ensure a node's connection to the multicast session delivery tree. AMRIS is an on demand protocol that constructs a shared delivery tree to support multiple senders and receivers within a multicast session. AMRIS dynamically assigns every node (on demand) in a multicast session with an ID number known as msm-id. The msm-id provides a heuristic height to a node and the ranking order of msm-id numbers directs the flow of datagram in the multicast delivery tree. Every node calculates its msm-id during the initialization phase, which is initiated by a special node called S-id. Normally, the S-id is the source node if there is only one source for the session. Otherwise, the S-id is the source node that has the minimum msm-id. The S-id broadcasts a NEW_SESSION message to its neighbours. When a node wants to join the multicast session, it chooses one of its neighbours which has the smaller msm-id as its parent and send it a JOIN-REQ message. If the neighbour is in the tree (if the tree has been built), it answers with a JOIN-ACK message, which means the joining is successful; otherwise (when it is the first time to build the tree), the neighbour forwards JOIN-REQ to its own neighbours and waits for the reply, which is repeated until the JOIN-REQ arrives at an on-tree node or the source. As a result, a delivery tree rooted from the source is formed to include all the group members and some relay non-members.

AMRIS repairs the broken links by performing local route repair without the need for any central controlling node, thereby reducing the control overhead.

\section{3) Core-Assisted Mesh protocol (CAMP)}

CAMP is designed to support multicast routing in highly

\section{Volume 4 Issue 11, November 2015}




\section{International Journal of Science and Research (IJSR) \\ ISSN (Online): 2319-7064}

Index Copernicus Value (2013): 6.14 | Impact Factor (2014): 5.611

dynamic ad-hoc networks [21,22]. It builds and maintains a multicast mesh between all sources and receivers in a multicast group. A shared multicast mesh is defined for each multicast group, and it is ensured that the shortest path between receivers and sources is part of the mesh. CAMP extends the receiver-initiated approach of the Core Based Tree (CBT) to create multicast meshes for MANETs. The CAMP protocol depends on a unicast protocol to provide topology information.

\section{B. Reactive Multicast Routing Protocols}

Traditional routing protocols such as On-Demand Multicast Routing Protocol (ODMRP) and Multicast Ad-hoc ondemand Distance Vector (MAODV) are Reactive multicast routing protocols. Reactive routing that means discovers the route when needed. Reactive routing protocols are well suited for a large-scale, narrow-band MANET with moderate or low mobility. Table 4 gives the Characteristic comparison of reactive Multicast Routing Protocol[4].

Table 4: Characteristics of Reactive Multicast Routing Protocol

\begin{tabular}{|c|c|c|}
\hline Characteristics & ODMRP & MAODV \\
\hline Multicast delivery structure & Mesh & Core based tree \\
\hline Loop free & Yes & Yes \\
\hline Periodic messages requirement & Yes & No \\
\hline Routing Hierarchy & Flat & Flat \\
\hline Scalability & Fair & Fair \\
\hline
\end{tabular}

1) On-Demand Multicast Routing Protocol (ODMRP) On-Demand Multicast Routing Protocol (ODMRP) [24] is a reactive mesh based multicast routing protocol. DMRP is not only a multicast routing protocol, but also provides unicast routing capability. The source establishes and maintains group membership and multicast mesh on demand if it needs to send data packets to the multicast group, which is somewhat similar to MAODV.ODMRP uses a forwarding group concept for multicast packet transmission, in which each multicast group $G$ is associated with a forwarding group (FG). Nodes in FG are in charge of forwarding multicast packets of group G. In a multicast group of ODMRP, the source manages the group membership, establishes and updates the multicast routes on demand.

\section{2) Multicast Ad-hoc On-demand Distance Vector (MAODV)}

Multicast operation of Ad-hoc On-demand Distance Vector (MAODV) is a reactive tree-based multicast routing protocol [23]. MAODV is an extension of the unicast routing protocol Ad-hoc On-demand Distance Vector (AODV). The main drawbacks of MAODV are long delays and high overheads associated with fixing broken links in conditions of high mobility and traffic load. Also, it has a low packet delivery ratio in scenarios with high mobility, large numbers of members, or a high traffic load. Because of its dependence on AODV, MAODV is not flexible. Finally, it suffers from a single point of failure, which is the multicast group leader.

\section{Hybrid Unicast Routing Protocols}

Hybrid routing protocol attempts to discover balance between the two such as proactive for neighborhood, reactive for far away. Based on proactive and reactive routing protocols, some hybrid routing protocols are proposed to combine their advantages. The most distinctive hybrid routing protocol is Zone Routing Protocol.

\section{1) Zone Routing Protocol (ZRP)}

Zone Routing Protocol (ZRP) [18] is a hybrid routing protocol for mobile ad hoc networks. The hybrid protocols are proposed to reduce the control overhead of proactive routing approaches and decrease the latency caused by route search operations in reactive routing approaches. Zone Routing Protocol (ZRP) [18] is a framework of hybrid routing protocol suites, which is made up the following modules: First one is Intra-zone Routing Protocol, second one is Inter-zone Routing Protocol, and last one is Bordercast Resolution Protocol.ZRP refers to the locally proactive routing component as the Intra-zone Routing Protocol (IARP). The globally reactive routing component is named Inter-zone Routing Protocol (IERP). IERP and IARP are not specific routing protocols. Instead, IARP is a family of limited-depth, proactive link-state routing protocols. IARP maintains routing information for nodes that are within the routing zone of the node. Correspondingly, IERP is a family of reactive routing protocols that offer enhanced route discovery and route maintenance services based on local connectivity monitored by IARP [27] [28].

\section{Hybrid Multicast Routing Protocols}

Traditional routing protocol such as Optimized Polymorphic Hybrid Multicast Routing Protocol (OPHMR) is the Hybrid multicast routing protocol. Hybrid routing protocol attempts to discover balance between the two such as proactive for neighbourhood, reactive for far away.

\section{1) Optimized Polymorphic Hybrid Multicast Routing protocol (OPHMR)}

OPHMR [25] protocol is invested with different operational modes that are either proactive or reactive based on a MN's power remainder, mobility level, and vicinity density level. It attempts to address the issues of power efficiency, latency, and protocol overhead in an adaptive manner. OPHMR's reactive behavior is based on the On-Demand Multicast Routing Protocol (ODMRP). It's relatively simplistic. It generates on-demand route paths for multicast message requests. OPHMR's proactive behavior is based on the Multicast Zone Routing (MZR) protocol. It builds a zone around each Mobile Node (in hops) and periodically sends updates within each defined zone. For added efficiency, OPHMR utilizes an optimizing scheme adapted from the Optimized Link State Routing (OLSR) protocol. It used to decrease the amount of control overhead that is produced. OPHMR is, after a very lengthy period of time, able to extend battery life and enhance the survivability of the mobile ad hoc nodes. As a result, it decreases the end-to-end delay and increases the packet delivery ratio.

\section{Conclusion}

MANET is an infra structure less IP based network which consists of mobile nodes and do not have any centralized administrator. To achieve best QoS , routing is an essential

\section{Volume 4 Issue 11, November 2015}




\section{International Journal of Science and Research (IJSR) \\ ISSN (Online): 2319-7064}

Index Copernicus Value (2013): 6.14 | Impact Factor (2014): 5.611

component of communication protocols in mobile ad hoc networks. Routing protocols typically fall under two classifications: first one is unicast routing protocol, second one is multicast routing protocol. The design of the protocols are driven by specific goals and requirements based on respective assumptions about the network properties or application area. On comparing these protocols Hybrid unicast and multicast routing protocols are better than proactive and reactive routing protocols. Multicasting communication serves as one critical operation to support many applications of mobile Ad hoc networks that achieve group communication rather than individual communication. Multicast routing protocols becomes increasingly important in MANETs because they effectively co-ordinate a set of nodes.

\section{References}

[1] Murthy, S. and J.J. Garcia-Luna-Aceves, -An Efficient Routing Protocol for Wireless Networks\|, ACM Mobile Networks and App. J., Special Issue on Routing in Mobile Communication Networks, Oct. 1996, pp. 18397.

[2] M. Ismail. - Routing Protocols for Ad Hoc Wireless Networks\|, M.Sc. (ISS) project, Carleton University, Ontario, Canada, August 2001

[3] J. N. Al-Karaki and A. E. Kamal, -Quality of Service Routing in Mobile Ad Hoc Networks: Current and Future Trends,\| Mobile Computing Handbook, I. Mahgoub and M. IIays, Eds., CRC Publishers, 2004.

[4] [4]http://www.csjournals.com/IJITKM/Special1/UNICA ST ANDMULTICAST.pdf

[5] C. R. Lin and J.-S. Liu, -QoS Routing in Ad Hoc Wireless Networks, IEEE JSAC, vol. 17, Aug. 1999, pp. 1426-38.

[6] S. Chen and K. Nahrstedt, -Distributed Quality-ofService Routing in Ad Hoc Networks, IEEE JSAC, vol. 17, Aug. 1999,pp. 1488-505.

[7] R. Bashandy, E. K. P. Chong, and A. Ghafoor, -Generalized Quality-of-Service Routing with Resource Allocation, IEEE JSAC, vol. 23, Feb. 2005, pp. 450-63.

[8] M. Wang and G.-S. Kuo, - An Application-Aware QoS Routing Scheme with Improved Stability for Multimedia Applications in Mobile Ad Hoc

[9] Networks, I Proc. IEEE Vehic. Tech. Conf., Sept. 2005, pp. 1901-05.

[10] Abdrabou and W. Zhuang, -A Position-Based QoS Routing Scheme for UWB Mobile Ad Hoc Networks, IEEE JSAC, vol.24, Apr. 2006, pp. 850-56.

[11]P. Jacquet, P. Muhlethaler, and A. Qayyum, -Optimized Link State Routing Protocoll, IETF MANET, Internet draft, 1998.

[12] Mario Gerla, Xiaoyan Hong, and Guangyu Pei, -Fisheye State Routing Protocol (FSR) for Ad Hoc Networks\|l, draft-ietf-manet-fsr-03.txt, June 2002.

[13] Richard G. Ogier, Fred L. Templin, Bhargav Bellur, and Mark G.Lewis, -Topology Broadcast Based on Reverse-Path Forwarding (TBRPF)\|, draft-ietf-manettbrpf-05.txt, March 2002.

[14] David B. Johnson, - Routing in Ad Hoc Networks of Mobile Hostsl, Proceedings of the IEEE workshop on Mobile Computing Systems and Applications, December
1994.

[15]D. Johnson, D. A. Maltz, —Dynamic source routing in ad hoc wireless networksl, in Mobile Computing ( $T$. Imielinski and H. Korth, eds.),Kluwer Acad. Publ., 1996.

[16]C.E. Perkins and E.M. Royer, -Ad hoc on demand Distance Vector routing\|, mobile computing systems and applications, 1999.Proceedings. WMCSA '99. Second IEEE Workshop on, 1999, p90 - p100 